\title{
Morbidity associated with 30-day surgical site infection following nonshunt pediatric neurosurgery
}

\author{
Brandon A. Sherrod, BS, and Brandon G. Rocque, MD, MS \\ Department of Neurosurgery, Division of Pediatric Neurosurgery, The University of Alabama at Birmingham and Children's \\ Hospital of Alabama, Birmingham, Alabama
}

\begin{abstract}
OBJECTIVE Morbidity associated with surgical site infection (SSI) following nonshunt pediatric neurosurgical procedures is poorly understood. The purpose of this study was to analyze acute morbidity and mortality associated with SSI after nonshunt pediatric neurosurgery using a nationwide cohort.

METHODS The authors reviewed data from the American College of Surgeons National Surgical Quality Improvement Program-Pediatric (NSQIP-P) 2012-2014 database, including all neurosurgical procedures performed on pediatric patients. Procedures were categorized by Current Procedural Terminology (CPT) codes. CSF shunts were excluded. Deep and superficial SSIs occurring within 30 days of an index procedure were identified. Deep SSls included deep wound infections, intracranial abscesses, meningitis, osteomyelitis, and ventriculitis. The following outcomes occurring within 30 days of an index procedure were analyzed, along with postoperative time to complication development: sepsis, wound disruption, length of postoperative stay, readmission, reoperation, and death.
\end{abstract}

RESULTS A total of 251 procedures associated with a 30 -day SSI were identified ( $2.7 \%$ of 9296 procedures). Superficial SSIs were more common than deep SSIs (57.4\% versus 42.6\%). Deep SSIs occurred more frequently after epilepsy or intracranial tumor procedures. Superficial SSIs occurred more frequently after skin lesion, spine, Chiari decompression, craniofacial, and myelomeningocele closure procedures. The mean $( \pm \mathrm{SD})$ postoperative length of stay for patients with any SSI was $9.6 \pm 14.8$ days (median 4 days). Post-SSI outcomes significantly associated with previous SSI included wound disruption (12.4\%), sepsis (15.5\%), readmission (36.7\%), and reoperation (43.4\%) ( $p<0.001$ for each). PostSSI sepsis rates $(6.3 \%$ vs $28.0 \%$ for superficial versus deep SSI, respectively; $p<0.001)$, wound disruption rates $(4.9 \%$ vs $22.4 \%, p<0.001)$, and reoperation rates $(23.6 \%$ vs $70.1 \%, p<0.001)$ were significantly greater for patients with deep SSIs. Postoperative length of stay in patients discharged before SSI development was not significantly different for deep versus superficial SSI (4.2 \pm 2.7 vs $3.6 \pm 2.4$ days, $p=0.094)$. No patient with SSI died within 30 days after surgery.

CONCLUSIONS Thirty-day SSI is associated with significant 30-day morbidity in pediatric patients undergoing nonshunt neurosurgery. Rates of SSI-associated complications are significantly lower in patients with superficial infection than in those with deep infection. There were no cases of SSI-related mortality within 30 days of the index procedure.

https://thejns.org/doi/abs/10.3171/2016.11.PEDS16455

KEY WORDS surgical site infection; morbidity; mortality; NSQIP; complication; pediatric neurosurgery

$\mathrm{H}$ OSPITAL-ACQUIRED infections are an increasingly prevalent and costly problem. ${ }^{28}$ Surgical site infection (SSI) is a hospital-acquired infection causing significant morbidity, mortality, and added cost of care. ${ }^{15,19,22,28}$ Although SSI itself is considered an undesired surgical complication, subsequent SSI-related adverse events such as bloodstream infection, septic shock, readmission, or reoperation are potentially more harmful than the SSI itself. The gravity of SSI, especially when consid- ering the potential preventability of the infection, should be understood in the appropriate context of its later consequences.

In pediatric neurosurgery, significant attention has been paid to infection following cerebrospinal fluid (CSF) shunt procedures. 13,14,26,27 However, much less attention has been paid to SSI or SSI-associated morbidity and mortality following nonshunt neurosurgical procedures. Moreover, although several studies have investigated SSI after non-

ABBREVIATIONS ACS = American College of Surgeons; HIPAA = Health Insurance Portability and Accountability Act; MMC = myelomeningocele; NSQIP = National Surgical Quality Improvement Program; NSQIP-P = NSQIP-Pediatric; SSI = surgical site infection; UTI = urinary tract infection.

SUBMITTED August 6, 2016. ACCEPTED November 9, 2016.

INCLUDE WHEN CITING Published online February 10, 2017; DOI: 10.3171/2016.11.PEDS16455. 
shunt pediatric neurosurgery at single centers, ${ }^{8,9,18,21,30,32}$ no studies have investigated multicenter nonshunt SSI outcomes. Due to potential variation in SSI rates by center and/or surgeon, analysis of multicenter data on SSI and its associated morbidity is needed. Furthermore, comparison of rates of SSI-associated complications by severity of SSI (e.g., deep or superficial infection) have been performed previously, although not specifically in pediatric neurosurgery. ${ }^{7,16}$

The primary goal of the current study was to review the associated acute morbidity of 30-day SSI following nonshunt pediatric neurosurgical procedures using a nationwide, validated, multicenter data set. A secondary goal of this study was to compare rates of SSI-associated complications by SSI subtype (deep/organ space vs superficial).

\section{Methods \\ Data Source and Reliability}

Our previous work has described the data source and its reliability in detail. ${ }^{24,25}$ Briefly, the American College of Surgeons National Surgical Quality Improvement Program-Pediatric (ACS NSQIP-P) is a clinical patient database comprised of surgical case from over 50 children's hospitals primarily in the United States. ${ }^{2-4}$ Each NSQIP-P site has an ACS-trained and -certified data abstractor; interrater disagreement rates are approximately $2 \%$ according to an ACS audit. ${ }^{2-4}$ Cases are sampled randomly on alternating 8-day cycles to ensure a random case distribution and to limit data abstractor workload. For more details regarding NSQIP-P, please reference the ACS NSQIP-P user guide files. ${ }^{2-4}$

The institutional review board (IRB) of the University of Alabama at Birmingham does not require IRB approval for mining the NSQIP-P data set because NSQIP-P data are deidentified and HIPAA (Health Insurance Portability and Accountability Act) compliant. ${ }^{29}$ For additional patient privacy protection, facility identifiers are not included in the NSQIP-P data set.

\section{Data Acquisition and Variables of Interest}

We used similar methodology to our previous work for data acquisition. ${ }^{24,25}$ Briefly, the 2012, 2013, and 2014 ACS NSQIP-P participant use files were queried for patients treated by a pediatric neurosurgeon. Procedures were categorized by Current Procedural Terminology (CPT) code into the following categories: spine, craniotomy for neoplasm, Chiari decompression, craniosynostosis, myelomeningocele (MMC) closure, epilepsy, skin lesion, and other (baclofen pump placement or removal procedures comprised more than $80 \%$ of this category). This categorization method is similar to that used in our previous work. ${ }^{24,25}$ Epilepsy procedures were not included in NSQIP-P until 2014. Shunt/ventricular catheter procedures were excluded from the analysis. Trauma cases (e.g., hematoma evacuation), transplantation cases, and cases with patients younger than 18 years at the time of the procedure are not included in NSQIP-P.

Surgical site infection in NSQIP-P is coded as 3 different subtypes according to CDC definitions: superficial incisional SSI, deep incisional SSI, and organ/space SSI. ${ }^{17}$
Superficial SSI is coded in NSQIP-P if any of the following is true: the superficial area of the surgical wound has purulent drainage, organisms are isolated from an aseptically obtained wound culture, one or more of the signs or symptoms of superficial SSI are present (pain/tenderness, localized swelling, erythema, heat; not a valid criterion if wound cultures are negative), or SSI is diagnosed by an attending physician or surgeon. Deep SSI is coded in NSQIP if at least one of the following is true: the deep area of the surgical wound has purulent drainage (wounds with both superficial and deep drainage are coded as deep SSI); an abscess of the deep surgical wound space is found by radiographic imaging modalities, physical examination of the surgical wound, reoperation for wound exploration or debridement, or by histopathologic modalities; one or more of the signs/symptoms of deep SSI are present with concurrent spontaneous wound dehiscence (fever $>38^{\circ} \mathrm{C}$, localized pain, tenderness; is not a valid criterion if wound cultures are negative); or SSI is diagnosed by an attending physician or surgeon. Organ/space SSI is coded in NSQIP$\mathrm{P}$ if any of the following is true: purulent drainage is documented coming from a stab wound to the operative organ/ space, organisms are isolated from an aseptically obtained fluid or tissue culture of the organ/space, or organ/space SSI is diagnosed by an attending physician or surgeon. Organ/space SSIs relevant to neurosurgery that are explicitly mentioned in the NSQIP-P wound occurrences criteria include osteomyelitis, intracranial abscess, dural abscess, meningitis, ventriculitis, and spinal abscess. For more information regarding NSQIP-P SSI coding criteria, please refer to the NSQIP-P user guide files..$^{2-4}$ SSI subtypes were combined into a single categorical outcome variable (any SSI) and then further categorized into deep or superficial infection. Deep SSI and organ/space SSI were combined into one category (deep SSI) due to the unclear distinction between the two in neurosurgery and the high likelihood that NSQIP-P interrater agreement rates between deep and organ/space SSI for neurosurgery cases are variable.

Postoperative outcomes were tracked for 30 postoperative days and included urinary tract infection (UTI), pneumonia, bleeding requiring transfusion, unplanned reintubation, sepsis, wound disruption, readmission, reoperation, and death. Data on days to complication were also analyzed. For sepsis/septic shock/SIRS (systemic inflammatory response syndrome) to be coded, criteria for both pediatric SIRS and suspected/proven infection must be met. More specifically, at least one the following criteria must be met: abnormal body temperature $\left(>38^{\circ} \mathrm{C}\right.$ or $<$ $36^{\circ} \mathrm{C}$ ), persistent tachycardia (or bradycardia for children younger than 1 year), persistent tachypnea, leukopenia or leukocytosis, and suspected or proven infection. Abnormal body temperature or leukocytosis and suspected/ proven infection must be present for sepsis to be coded. Septic shock was also included under sepsis and was defined as systemic sepsis (see previously listed criteria) with accompanying documented cardiovascular dysfunction. Preoperative sepsis was not included in postoperative sepsis coding unless a newly identified source of infection was identified postoperatively. For more information regarding NSQIP-P complication coding criteria, please refer to the NSQIP-P user guide files. ${ }^{2-4}$ 
TABLE 1. Distribution of SSIs by procedure category and SSI subtype in NSQIP.P

\begin{tabular}{lcrcr}
\hline & No. of & \multicolumn{3}{c}{ No. of SSIs (\%) } \\
\cline { 3 - 5 } & $\begin{array}{c}\text { Procedures } \\
\text { Procedure Category }\end{array}$ (\% of total) & All SSIs & Deep & Superficial \\
\hline Spine & $2879(31)$ & $101(3.5)$ & $25(24.8)$ & $76(75.2)$ \\
\hline $\begin{array}{l}\text { Craniotomy for } \\
\text { neoplasm }\end{array}$ & $2434(26)$ & $60(2.5)$ & $43(71.7)$ & $17(28.3)$ \\
\hline Chiari decompression & $1748(19)$ & $33(1.9)$ & $22(33.3)$ & $11(66.7)$ \\
\hline $\begin{array}{l}\text { Craniosynostosis/ } \\
\text { craniofacial }\end{array}$ & $980(11)$ & $15(1.5)$ & $6(40.0)$ & $9(60.0)$ \\
\hline MMC closure & $368(4)$ & $16(4.4)$ & $7(43.7)$ & $9(56.3)$ \\
\hline Epilepsy* & $208(2)$ & $7(3.4)$ & $6(85.7)$ & $1(14.3)$ \\
\hline Skin lesion & $136(2)$ & $1(0.7)$ & $0(0)$ & $1(100)$ \\
\hline Other† & $543(6)$ & $18(3.3)$ & $9(50.0)$ & $9(50.0)$ \\
\hline Total & 9296 & $251(2.7)$ & $107(42.6)$ & $144(57.4)$ \\
\hline
\end{tabular}

* Epilepsy procedures were not added to NSQIP-P until 2014.

† Other procedures are primarily baclofen pump procedures (> $80 \%$ within this category).

For time to event analysis, NSQIP-P data abstractors code the number of days to the SSI event by entering the earliest date at which one of the above SSI criteria was met. Time to postoperative outcomes is coded in a similar manner. For the purposes of this study, in which we seek to examine the consequences of SSI, infection was considered as occurring before or concurrent with the postoperative complication if time to SSI was less than or equal to the time to postoperative complications.

\section{Statistical Analysis}

NSQIP-P 30-day outcome variables were compared to determine association with any postoperative SSI occurrence, and also with deep or superficial SSI, via a chi-square test, an independent-samples t-test, or a MannWhitney U-test where appropriate. Contingency tables were created for all categorical variables to calculate relative SSI frequencies with subsequent calculation of corresponding odds ratios and $95 \%$ confidence intervals.
Variables were considered significant when $\mathrm{p}<0.05$. Statistical analysis was performed using SPSS version 23.0 (IBM Corporation).

\section{Results}

A total of 9296 nonshunt neurosurgical procedures performed on pediatric patients from 2012 to 2014 were identified in NSQIP-P. The overall 30-day SSI rate was $2.7 \%$. The mean length of time between the index procedure and SSI development was $14.6 \pm 6.8$ days $( \pm \mathrm{SD})$.

\section{Procedural SSI Frequency}

The distribution of SSIs by procedure category is displayed in Table 1, with additional data on deep versus superficial SSI rates by procedure category. Spine procedures were the most common procedure in the NSQIP-P neurosurgical cohort (31\% of all cases) followed by craniotomies for neoplasms (26\%) and Chiari malformation (19\%). Superficial SSIs occurred more frequently than deep SSIs overall (57.4\% vs 42.6\%). Deep SSIs were more likely after procedures for epilepsy or intracranial tumors. Superficial SSIs were more likely after skin lesion, spine, Chiari decompression, craniofacial, and MMC closure procedures.

\section{Adverse Outcomes for Any SSI Occurrence}

Table 2 contains data on adverse outcomes associated with any 30-day SSI. Outcomes that occurred after SSI and were significantly associated with SSI included wound disruption (12.4\% in patients with SSI vs $1.1 \%$ in those without SSI), sepsis (15.5\% vs $0.3 \%$ ), hospital stay longer than 30 postoperative days ( $7.2 \%$ vs $1.2 \%)$, readmission within 30 days after the index procedure $(36.7 \%$ vs $8.4 \%$ ), and reoperation within 30 days of the index procedure $(43.4 \%$ vs $5.2 \%)$. There were no recorded deaths at 30 postoperative days in patients with SSI. The effect size was greatest for sepsis (OR 61.4) and reoperation (OR 13.9) for rates of complications in patients with SSI versus patients without SSI. Time from SSI to complication is shown, with most complications occurring within approximately 1-2 days after SSI was documented; the

TABLE 2. Postoperative adverse outcomes associated with any 30-day SSI

\begin{tabular}{lcccccc}
\hline \multicolumn{1}{c}{ Outcome } & $\begin{array}{c}\mathrm{SSI} \\
\mathrm{n}=251)\end{array}$ & $\begin{array}{c}\text { No SSI } \\
(\mathrm{n}=9045)\end{array}$ & $\begin{array}{c}\text { Mean Time from SSI } \\
\text { to Complication (days) }\end{array}$ & OR & $\begin{array}{c}\text { 95\% Cl } \\
\text { Value }\end{array}$ \\
\hline Wound disruption & $31(12.4)$ & $103(1.1)$ & $0.9 \pm 3.3$ & 12.233 & $8.012-18.678$ & $<0.001$ \\
\hline Sepsis & $39(15.5)$ & $27(0.3)$ & $0.7 \pm 2.0$ & 61.443 & $36.925-102.243$ & $<0.001$ \\
\hline UTI & $4(1.6)$ & $95(1.1)$ & $7.0 \pm 12.7$ & 1.526 & $0.557-4.182$ & 0.408 \\
\hline Unplanned reintubation & $2(0.8)$ & $73(0.8)$ & $2.0 \pm 2.8$ & 0.987 & $0.241-4.045$ & 0.986 \\
\hline Death & $0(0)$ & $26(0.3)$ & $\mathrm{NA}$ & $\mathrm{NA}$ & & \\
\hline Readmission w/in 30 days postop & $92(36.7)$ & $761(8.4)$ & $1.4 \pm 4.1$ & 6.299 & $4.821-8.228$ & $<0.001$ \\
\hline Reoperation w/in 30 days postop & $109(43.4)$ & $473(5.2)$ & $1.7 \pm 3.7$ & 13.911 & $10.660-18.154$ & $<0.001$ \\
\hline Postop LOS >30 days & $18(7.2)$ & $111(1.2)$ & $\mathrm{NA}$ & 6.218 & $3.716-10.404$ & $<0.001$ \\
\hline
\end{tabular}

LOS = length of stay in hospital; NA = not available.

Data are presented as numbers of cases (\%) unless otherwise indicated. Means are presented with SDs. Outcomes were excluded from the SSI group if they occurred before SSI (except postop LOS $>30$ days). Statistical analysis was performed using the chi-square test and Fisher exact test where appropriate. Boldface type indicates statistical significance $(p<0.05)$. 
TABLE 3. Postoperative adverse outcomes associated with deep versus superficial 30 -day SSI

\begin{tabular}{lccccc}
\hline \multicolumn{1}{c}{ Outcome } & Deep SSI & Superficial SSI & OR $^{*}$ & $95 \% \mathrm{Cl}$ & $\mathrm{p} \mathrm{Value}$ \\
\hline Wound disruption & $24(22.4)$ & $7(4.9)$ & 5.659 & $2.336-13.712$ & $<0.001$ \\
\hline Sepsis & $30(28.0)$ & $9(6.3)$ & 5.844 & $2.637-12.951$ & $<0.001$ \\
\hline UTI & $2(1.9)$ & $2(1.4)$ & 1.352 & $0.187-9.757$ & 0.764 \\
\hline Unplanned reintubation & $2(1.9)$ & $0(0)$ & $\mathrm{NA}$ & & \\
\hline Death & $0(0)$ & $0(0)$ & $\mathrm{NA}$ & & \\
\hline Readmission w/in 30 days & $41(38.3)$ & $51(35.4)$ & 1.133 & $0.675-1.902$ & 0.637 \\
\hline Reoperation w/in 30 days & $75(70.1)$ & $34(23.6)$ & 7.583 & $4.310-13.341$ & $<0.001$ \\
\hline Postop LOS $>$ 30 days & $12(11.2)$ & $6(4.2)$ & 2.905 & $1.054-8.010$ & $\mathbf{0 . 0 4 6}$ \\
\hline
\end{tabular}

Outcomes were excluded if they occurred before SSI (except postop LOS $>30$ days). Data are presented as number of cases (\%). Statistical analysis was performed using the chi-square test and Fisher exact test where appropriate. Boldface type indicates statistical significance $(p<$ 0.05).

* Odds of a patient with deep SSI experiencing the outcome of interest relative to patient with superficial SSI.

exception was UTI, which occurred at a mean of 7 days post-SSI.

\section{Adverse Outcomes for Deep Versus Superficial SSI}

Table 3 displays adverse outcomes for deep versus superficial 30-day SSI. Rates of wound disruption (22.4\% for deep SSI vs $4.9 \%$ for superficial), sepsis (28.0\% vs $6.3 \%$ ), reoperation $(70.1 \%$ vs $23.6 \%$ ), and hospital stay $>$ 30 days (11.2\% vs $4.2 \%)$ were all significantly greater in patients with deep SSI than in those with superficial SSI. No significant difference was observed for UTI or readmission. No single adverse outcome occurred with greater frequency in the superficial SSI group. Of all outcomes, reoperation showed the greatest difference in effect size for deep versus superficial SSI (OR 7.6).

\section{Time to SSI Occurrence}

Figure 1 displays data on postoperative time to SSI (Fig. 1 left) and postoperative length of stay by SSI subtype (Fig. 1 right). There was no significant difference between deep and superficial SSIs with respect to time to SSI occurrence $(p=0.492)$. The postoperative length of stay was significantly longer for patients with deep SSIs than for those with superficial SSIs. Patients with any SSI had a significantly longer postoperative length of stay than patients without SSI $(9.6 \pm 14.8$ days vs $4.7 \pm 6.8$ days, $\mathrm{p}<$ $0.001)$. There was significant variation in length of stay for patients with deep SSIs in particular, with an interquartile range of 3-21 days. The postoperative length of stay for patients discharged before SSI development, however, did not differ significantly between those with deep SSIs and those with superficial SSIs ( $4.2 \pm 2.7$ vs $3.6 \pm 2.4$ days, $p$ $=0.094)$.

\section{SSI Trends}

Figure 2 displays data on absolute and relative rates of deep and superficial SSI over time. No significant differences were observed between years for absolute or relative rates of SSI. Superficial SSI rates were greater than deep SSI rates for all years analyzed.

\section{Discussion}

Prior to this study, morbidity and mortality of nonshunt
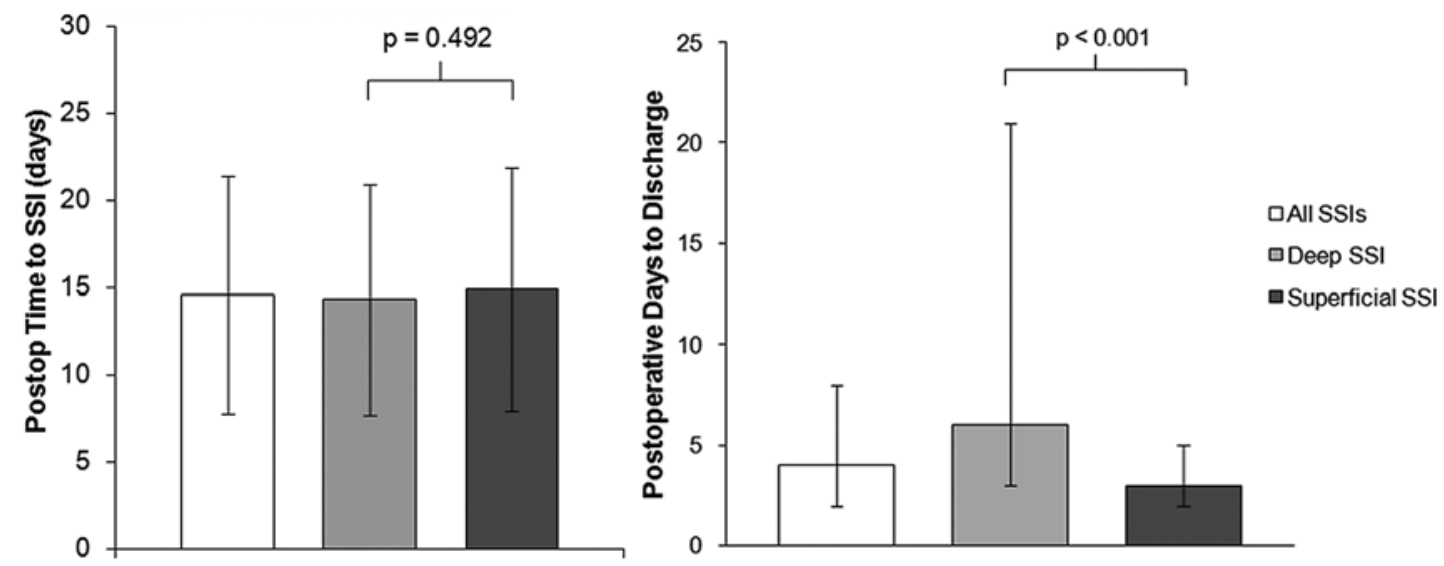

FIG. 1. Left: Postoperative time to SSI event by SSI type. Data are expressed as mean \pm SD (error bars). No significant differences were observed for superficial versus deep SSI by 1-way ANOVA. Right: Postoperative time to discharge by SSI type. Data are expressed as median and interquartile range (error bars). Statistical tests were performed using both 1-way ANOVA and Mann-Whitney U-test $(p<0.001$ for both tests). 


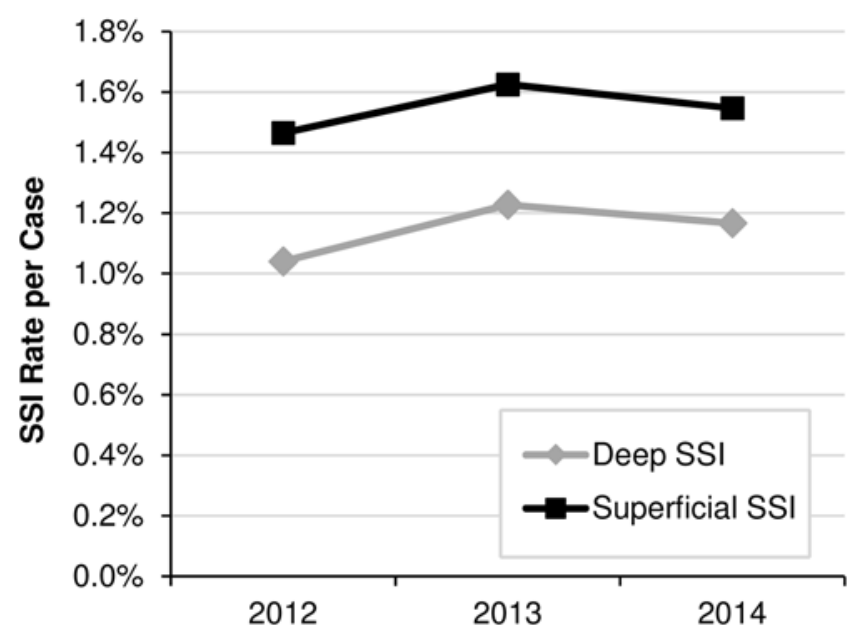

FIG. 2. Trends in NSQIP-P deep and superficial SSI rates for 2012-2014.

SSI in pediatric neurosurgery have not been examined in detail. We have identified rates of morbidity and mortality in patients with documented 30-day SSI following nonshunt pediatric neurosurgical procedures using a national clinical database. Thirty-day SSI is associated with significant 30-day morbidity in pediatric patients undergoing nonshunt neurosurgery. Rates of SSI-associated complications are significantly lower in patients with superficial infection than in those with deep infection. There were no cases of SSI-related mortality within 30 days of the index procedure.

\section{SSI-Associated Morbidity}

We observed significant morbidity in patients with SSIs, particularly in those with deep SSIs. For patients with any SSI, rates of sepsis, wound disruption, readmission, and reoperation were significantly greater than for patients without SSI. Furthermore, length of postoperative stay was significantly longer in patients with any SSI. These findings indicate that SSI is associated with significant acute morbidity and cost of care.

Rates of most complications were significantly greater for patients experiencing deep SSI than for those with superficial SSI. This finding is unsurprising given the relative severity of deep SSI and has been reported in previous studies. ${ }^{7}$ Importantly, with the exception of epilepsy or intracranial tumor procedures, most nonshunt procedures were more frequently associated with superficial SSI than with deep SSI. Therefore, patients undergoing epilepsy or intracranial tumor procedures should be monitored closely for deep infection and its associated complication. These patients may benefit most from efforts targeted at SSI prevention. Authors of future studies on SSI outcomes should be cautious in reporting complications for a single SSI categorical outcome given the differences in deep versus superficial SSI.,16

Sepsis was the most concerning medical complication of SSI, with SSI-related sepsis rates approaching 30\% in the deep SSI group. However, it is important to recognize that although sepsis was only analyzed if it occurred after SSI, this does not necessarily imply causality; sepsis could have occurred as the result of an unrelated, non-SSI.
Nevertheless, efforts focused toward SSI prevention, particularly for deep infection, could have a profound impact on subsequent systemic infection development. Although no patients with SSI died in this cohort within 30 postoperative days, sepsis is one of the leading causes of death in children worldwide. ${ }^{6}$ One study reported over 75,000 pediatric hospitalizations in 2005 involving severe sepsis, with an associated cost of $\$ 4.8$ billion. ${ }^{11}$ Pediatric sepsis, when severe, carries an average cost of more than $\$ 40,000$ per case and a mortality rate greater than $10 \% .5,31$ Furthermore, infections of the central nervous system have been shown to be second only to endocarditis for sepsisassociated mortality. ${ }^{31}$ While it is not possible to discern how many cases of severe pediatric sepsis were due to SSI, even a small fraction of the aforementioned cases might have been prevented if SSI had not occurred, potentially leading to reduced cost of care, morbidity, and length of stay.

\section{Return to System}

Return to system for reoperation or readmission occurred frequently for both categories of SSI, highlighting the resource-intensive nature of caring for an SSI. Patients with deep SSI had much higher odds of needing another operation (OR 7.6 for reoperation vs OR 1.1 for readmission). Our previous study on readmission risk factors in pediatric neurosurgery found that SSI was the strongest risk factor for unplanned readmission, and this current work provides more evidence for SSI as a potentially preventable cause of readmission. ${ }^{25}$ In one adult neurosurgical study, $73.9 \%$ of patients with SSI after undergoing surgery for intracranial tumors, chronic subdural hematomas, or reconstructive cranioplasty required reoperation. ${ }^{1}$ Another study found that the top reasons for reoperation and readmission after pediatric spinal fusion surgery at a single institution included wound dehiscence, deep wound infection, and superficial wound infection; these findings are in agreement with our own. ${ }^{12}$ Therefore, prevention of SSI could have a profound effect on reducing return to system after pediatric neurosurgery.

\section{Cost of Care}

Although the data in this study did not include cost of SSI treatment or subsequent care, it is important to discuss the implications of our data in the context of financial resources. We demonstrated a significant and substantial increase in rates of readmission, reoperation, and later complications for patients with SSI, particularly deep SSI. Furthermore, length of stay was substantially longer in SSI patients, with patients with deep SSIs having significantly longer postoperative length of stay than patients with superficial SSIs. Return to system, longer length of stay, and treatment for SSI-associated complications are expensive occurrences that could potentially be reduced with a reduction in SSI. One study showed that in 129 Veterans Affairs hospitals across the United States, adult neurosurgical patients had the highest SSI-related cost of care, with a risk-adjusted cost increase of $\$ 23,755$ above patients without SSI. ${ }^{22}$ Furthermore, the difference in riskadjusted cost for deep versus superficial SSIs was over 
$\$ 18,000$ per event. Importantly, costs do not only accrue during the initial hospital stay; one study found that the total costs 8 weeks after discharge for adult patients with SSI was over $\$ 3000$ more than costs for patients without SSI. ${ }^{20}$ The authors attributed these costs to more outpatient visits, emergency department visits, readmissions, and home health services. Future study is warranted to evaluate SSI-associated costs in pediatric neurosurgery specifically.

\section{Pertinent Negative Findings}

The major negative finding of this study is the lack of mortality in any patient with SSI at 30 postoperative days. This is an encouraging negative finding, potentially providing evidence that medical care for patients with SSI may be effectively managing the more severe SSI-related complications, such as sepsis. However, follow-up beyond 30 postoperative days is not tracked in NSQIP-P. Given that SSI does not become apparent until a mean of 14 days after surgery, severe adverse events may not have time to occur in the 30-day follow-up period.

\section{Study Limitations}

The primary limitation of this study is the lack of follow-up for SSI and SSI-associated complications beyond 30 postoperative days. The results of this study, particularly regarding rates of SSI and complications after surgery regardless of follow-up window, are likely skewed due to underreporting of complications occurring more than 30 days postoperatively. There are no data in NSQIP-P regarding severity of postoperative complications, making our analysis insufficiently granular for many complications. NSQIP$\mathrm{P}$ lacks information on specific subtype of deep SSI (e.g., meningitis, ventriculitis, intracranial abscess), causative organism, and culture site, all of which may affect the subsequent morbidity; previous studies have shown that different organisms cultured from SSIs result in different severity of morbidity. ${ }^{10}$ The NSQIP-P data set lacks any information on costs associated with postoperative treatment or followup care, which is important to consider when investigating a potentially preventable outcome such as SSI. However, the costs of SSI have been studied thoroughly., $20,22,23,28 \mathrm{Fa}-$ cility identifiers are excluded from NSQIP-P for HIPAA reasons, meaning that outlier facilities could disproportionately affect rates of SSI and SSI-associated complications. There is likely underreporting of clinical infections that lack documented positive cultures, signs/symptoms, or physician diagnosis since Gram stain results and other CSF laboratory results alone do not meet NSQIP-P criteria for SSI coding. Epilepsy procedures were not included in the 2012-2013 data sets, meaning that the relative procedure frequencies for all procedure categories are likely skewed. Because NSQIP-P cases are randomly sampled in 8-day cycles, there is potential for sampling error if a substantial number of SSIs occurred in nonsampled cases. ${ }^{2-4}$ NSQIP$\mathrm{P}$ excludes trauma cases, presumably excluding a relevant portion of neurosurgery cases (e.g., hematoma evacuation).

Despite these limitations, this study may help pediatric neurosurgeons identify acute complications associated with SSI and help surgeons understand the extent of nonshunt SSI burden. Furthermore, the results of this study may help identify procedures with higher rates of deep infection, which are more likely to result in serious complications.

\section{Conclusions}

We have identified rates of acute morbidity associated with 30-day SSI after nonshunt pediatric neurosurgery using a national database. Acute SSI-associated morbidity following nonshunt procedures is significant, particularly regarding sepsis, wound disruption, longer length of stay, readmission, and reoperation. Deep SSI occurs less frequently than superficial SSI, yet deep SSI has significantly greater rates of many complications. Patients undergoing procedures for intracranial tumors and epilepsy are more likely to experience deep SSI and are therefore more likely to experience serious SSI-related complications. There was no SSI-associated mortality at 30 postoperative days in our study cohort.

The results of this study should be interpreted with caution due to the insufficiency of 30-day follow-up for capturing all SSIs and SSI-related complications. Furthermore, the lack of data on severity of NSQIP-P complications limits the thoroughness of our results. Despite these limitations, the data in this study illuminate the burden of SSI in the nonshunt pediatric neurosurgical population. Acute complications and return to system are significant for SSI patients, particularly those experiencing deep SSI.

\section{Acknowledgments}

Dr. Rocque is supported by NIH Grant 1KL2TR001419 and by the Kaul Pediatric Research Institute of Children's of Alabama.

The ACS NSQIP and the hospitals participating in the ACS NSQIP-P are the source of the data used herein; they have not verified and are not responsible for the statistical validity of the data analysis or the conclusions derived by the authors.

\section{References}

1. Abu Hamdeh S, Lytsy B, Ronne-Engström E: Surgical site infections in standard neurosurgery procedures - a study of incidence, impact and potential risk factors. Br J Neurosurg 28:270-275, 2014

2. American College of Surgeons: User Guide for the 2012 ACS NSQIP Pediatric Participant Use Data File. Chicago: American College of Surgeons, 2013. (https://www.facs.org/ /media/files/quality\%20programs/ nsqip/2012pedsuserguide.ashx) [Accessed December 15, 2016]

3. American College of Surgeons: User Guide for the 2013 ACS NSQIP Pediatric Participant Use Data File. Chicago: American College of Surgeons, 2014. (https://www.facs. org/ /media/files/quality\%20programs/nsqip/peds_puf_ userguide_2013.ashx) [Accessed December 15, 2016]

4. American College of Surgeons: User Guide for the 2014 ACS NSQIP Pediatric Participant Use Data File. Chicago: American College of Surgeons, 2015. (https://www.facs. org/ /media/files/quality\%20programs/nsqip/peds_acs_ nsqip_puf_userguide_2014.ashx) [Accessed December 15, 2016]

5. Angus DC, Linde-Zwirble WT, Lidicker J, Clermont G, Carcillo J, Pinsky MR: Epidemiology of severe sepsis in the United States: analysis of incidence, outcome, and associated costs of care. Crit Care Med 29:1303-1310, 2001 
6. Black RE, Cousens S, Johnson HL, Lawn JE, Rudan I, Bassani DG, et al: Global, regional, and national causes of child mortality in 2008: a systematic analysis. Lancet 375:19691987, 2010

7. Coello R, Charlett A, Wilson J, Ward V, Pearson A, Borriello P: Adverse impact of surgical site infections in English hospitals. J Hosp Infect 60:93-103, 2005

8. Demir N, Peker E, Gülșen I, Ağengin K, Tuncer O: Factors affecting infection development after meningomyelocele repair in newborns and the efficacy of antibiotic prophylaxis. Childs Nerv Syst 31:1355-1359, 2015

9. Elward A, Yegge J, Recktenwald A, Jadwisiak L, Kieffer P, Hohrein M, et al: Risk factors for craniotomy or spinal fusion surgical site infection. Pediatr Infect Dis J 34:1323-1328, 2015

10. Engemann JJ, Carmeli Y, Cosgrove SE, Fowler VG, Bronstein MZ, Trivette SL, et al: Adverse clinical and economic outcomes attributable to methicillin resistance among patients with Staphylococcus aureus surgical site infection. Clin Infect Dis 36:592-598, 2003

11. Hartman ME, Linde-Zwirble WT, Angus DC, Watson RS: Trends in the epidemiology of pediatric severe sepsis. Pediatr Crit Care Med 14:686-693, 2013

12. Jain A, Puvanesarajah V, Menga EN, Sponseller PD: Unplanned hospital readmissions and reoperations after pediatric spinal fusion surgery. Spine (Phila Pa 1976) 40:856-862, 2015

13. Kestle JR, Holubkov R, Cochrane DD, Kulkarni AV, Limbrick DD Jr, Luerssen TG, et al: A new Hydrocephalus Clinical Research Network protocol to reduce cerebrospinal fluid shunt infection. J Neurosurg Pediatr 17:391-396, 2016

14. Kestle JR, Riva-Cambrin J, Wellons JC III, Kulkarni AV, Whitehead WE, Walker ML, et al: A standardized protocol to reduce cerebrospinal fluid shunt infection: the Hydrocephalus Clinical Research Network Quality Improvement Initiative. J Neurosurg Pediatr 8:22-29, 2011

15. Kirkland KB, Briggs JP, Trivette SL, Wilkinson WE, Sexton DJ: The impact of surgical-site infections in the 1990s: attributable mortality, excess length of hospitalization, and extra costs. Infect Control Hosp Epidemiol 20:725-730, 1999

16. Lawson EH, Hall BL, Ko CY: Risk factors for superficial vs deep/organ-space surgical site infections: implications for quality improvement initiatives. JAMA Surg 148:849-858, 2013

17. Mangram AJ, Horan TC, Pearson ML, Silver LC, Jarvis WR: Guideline for prevention of surgical site infection, 1999. Am J Infect Control 27:96-134, 1999

18. Meng F, Cao J, Meng X: Risk factors for surgical site infection following pediatric spinal deformity surgery: a systematic review and meta-analysis. Childs Nerv Syst 31:521-527, 2015

19. Owens CD, Stoessel K: Surgical site infections: epidemiology, microbiology and prevention. J Hosp Infect 70 (Suppl 2):3-10, 2008

20. Perencevich EN, Sands KE, Cosgrove SE, Guadagnoli E, Meara E, Platt R: Health and economic impact of surgical site infections diagnosed after hospital discharge. Emerg Infect Dis 9:196-203, 2003

21. Ryan SL, Sen A, Staggers K, Luerssen TG, Jea A: A standardized protocol to reduce pediatric spine surgery infection: a quality improvement initiative. J Neurosurg Pediatr 14:259-265, 2014

22. Schweizer ML, Cullen JJ, Perencevich EN, Vaughan Sarrazin MS: Costs associated with surgical site infections in Veterans Affairs hospitals. JAMA Surg 149:575-581, 2014

23. Shepard J, Ward W, Milstone A, Carlson T, Frederick J, Hadhazy E, et al: Financial impact of surgical site infections on hospitals: the hospital management perspective. JAMA Surg 148:907-914, 2013
24. Sherrod BA, Arynchyna AA, Johnston JM, Rozzelle CJ, Blount JP, Oakes WJ, et al: Risk factors for surgical site infection following nonshunt pediatric neurosurgery: a review of 9296 procedures from a national database and comparison with a single-center experience. J Neurosurg Pediatr [epub ahead of print February 10, 2017. DOI: 10.3171/2016.11. PEDS16454]

25. Sherrod BA, Johnston JM, Rocque BG: Risk factors for unplanned readmission within 30 days after pediatric neurosurgery: a nationwide analysis of 9799 procedures from the American College of Surgeons National Surgical Quality Improvement Program. J Neurosurg Pediatr 18:350-362, 2016

26. Simon TD, Butler J, Whitlock KB, Browd SR, Holubkov R, Kestle JR, et al: Risk factors for first cerebrospinal fluid shunt infection: findings from a multi-center prospective cohort study. J Pediatr 164:1462-1468, 1468.e1-1468.e2, 2014

27. Simon TD, Hall M, Riva-Cambrin J, Albert JE, Jeffries HE, Lafleur B, et al: Infection rates following initial cerebrospinal fluid shunt placement across pediatric hospitals in the United States. J Neurosurg Pediatr 4:156-165, 2009

28. Stone PW, Braccia D, Larson E: Systematic review of economic analyses of health care-associated infections. Am J Infect Control 33:501-509, 2005

29. University of Alabama at Birmingham Institutional Review Board: Frequently asked questions: Is IRB review required for use of public datasets? UAB Research. (http://www.uab. edu/research/administration/offices/IRB/FAQs/Pages/default. aspx?Topic $=$ Datasets) [Accessed December 15, 2016]

30. von Lehe M, Kim HJ, Schramm J, Simon M: A comprehensive analysis of early outcomes and complication rates after 769 craniotomies in pediatric patients. Childs Nerv Syst 29:781-790, 2013

31. Watson RS, Carcillo JA, Linde-Zwirble WT, Clermont G, Lidicker J, Angus DC: The epidemiology of severe sepsis in children in the United States. Am J Respir Crit Care Med 167:695-701, 2003

32. Yeung LC, Cunningham ML, Allpress AL, Gruss JS, Ellenbogen RG, Zerr DM: Surgical site infections after pediatric intracranial surgery for craniofacial malformations: frequency and risk factors. Neurosurgery 56:733-739, 2005

\section{Disclosures}

The authors report no conflict of interest concerning the materials or methods used in this study or the findings specified in this paper.

\section{Author Contributions}

Conception and design: both authors. Acquisition of data: Sherrod. Analysis and interpretation of data: both authors. Drafting the article: both authors. Critically revising the article: both authors. Reviewed submitted version of manuscript: both authors. Approved the final version of the manuscript on behalf of both authors: Rocque. Statistical analysis: both authors. Study supervision: Rocque.

\section{Supplemental Information}

\section{Previous Presentations}

This paper was presented on December 7, 2016, at the 2016 Annual Meeting of the AANS/CNS Section on Pediatric Neurological Surgery, Orlando, FL.

\section{Correspondence}

Brandon G. Rocque, Department of Neurosurgery, Division of Pediatric Neurosurgery, The University of Alabama at Birmingham, 1600 7th Ave. South, Lowder 400, Birmingham, AL 35233 5294.email: brandon.rocque@childrensal.org. 